\title{
A Mobile Robot for Locomotion through a 3D Periodic Lattice Environment
}

\author{
Benjamin Jenett \\ Center for Bits and Atoms \\ MIT, Cambridge, MA \\ bej@mit.edu
}

\author{
Daniel Cellucci \\ Department of Mechanical and \\ Aerospace Engineering \\ Cornell University, Ithaca, NY \\ dwc238@cornell.edu
}

\author{
Kenneth Cheung \\ NASA Ames Research Center \\ Moffett Field, CA \\ kenny@nasa.gov
}

\begin{abstract}
This paper describes a novel class of robots specifically adapted to climb periodic lattices, which we call "Relative Robots". These robots use the regularity of the structure to simplify the path planning, align with minimal feedback, and reduce the number of degrees of freedom (DOF) required to locomote. They can perform vital inspection and repair tasks within the structure that larger truss construction robots could not perform without modifying the structure. We detail a specific type of relative robot designed to traverse a cuboctahedral (CubOct) cellular solids lattice, show how the symmetries of the lattice simplify the design, and test these design methodologies with a CubOct relative robot that traverses a $76.2 \mathrm{~mm}(3 \mathrm{in}$.) pitch lattice, MOJO (Multi-Objective JOurneying robot). We perform three locomotion tasks with MOJO: vertical climbing, horizontal climbing, and turning, and find that, due to changes in the orientation of the robot relative to the gravity vector, the success rate of vertical and horizontal climbing is significantly different.
\end{abstract}

\section{INTRODUCTION}

Reversibly-assembled digital cellular solids are a recent innovation in materials design. They consist of a threedimensional framework that has been decomposed into many identical building blocks that are then assembled together with a reversible mechanical connection to form materials with many desirable traits, including repairability, reconfigurability, and customizable anisotropic mechanical properties [1].

These solids have been demonstrated in aerospace applications such as morphing aircraft [2] and reconfigurable meter-scale structures [3]. Additionally, since the building blocks that compose the system have identical interfaces and similar dimensions, assembling these parts into the cellular solid has been identified as a process that would be straightforward to automate [4] [5].

$\mathrm{W}$ a robot that is designed to traverse and inspect the CubOct digital cellular solids lattice (Figure 1). While this robot draws from a long lineage of truss climbing robotic platforms, it is uniquely adapted to the periodic lattice geometry through which it moves. These adaptations allow it to simplify its path planning, align with minimal feedback, and locomote with fewer DOFs than other truss traversal platforms. This adaptation is a different enough approach to automated traversal that it describes a novel class of robotic systems, called "Relative Robots"

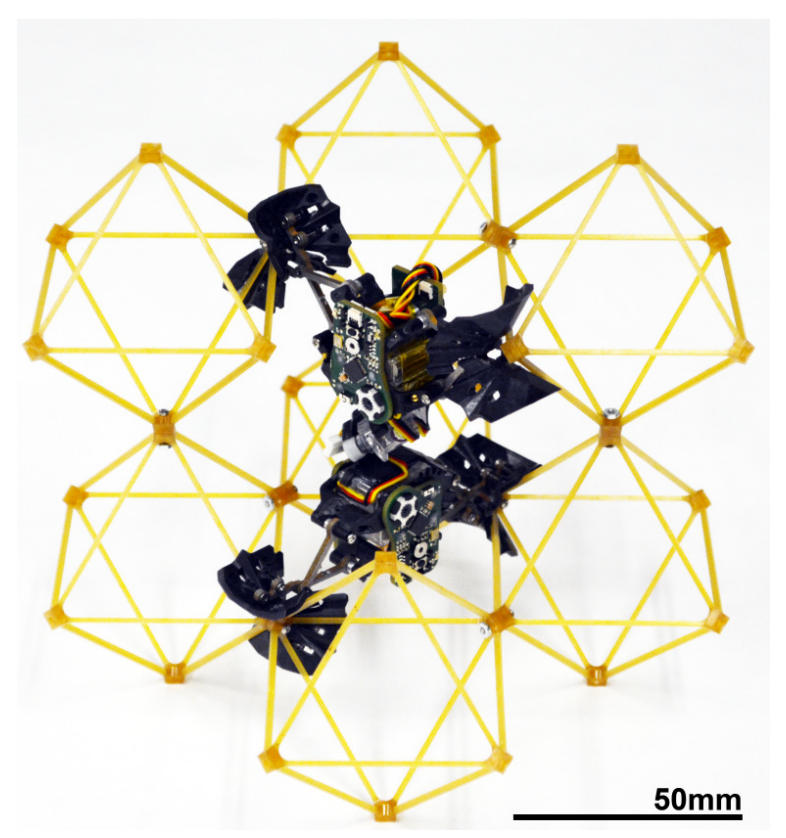

Figure 1: Multi-Objective JOurneying robot (MOJO). Robot is shown in lattice structure.

\section{BACKGROUND}

Framework construction robots perform a task similar to digital cellular solid assembly. These robots c consist of a multiple DOF manipulator mounted on a gantry or other locomotion platform, which assembles a structure from frame elements and nodes [6] [7] [8]. These robots have been proposed as constructors in hazardous or remote environments, such as space or deep underwater [8].

Dedicated robots capable of traversing the assembled structure and performing inspection or repair has been identified as a critical component of these sorts of systems [8]. This is because the construction robots mentioned above are mounted on the outside of the structure, and can only make changes to its surface. Performing a repair on an element in the middle of a volume of assembled structure would require disassembly of the entire section of the structure separating this failed element from the surface of that structure.

Research supported by the National Aeronautics and Space Administration (NASA) Aeronautics Research Institute Team Seedling Program, the NASA Convergent Aeronautics Solutions Program, and the NASA Space Technology Research Fellowship (NSTRF) Program. 

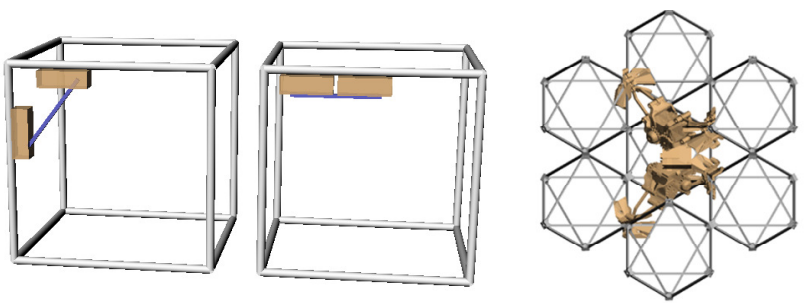

Figure 2: Comparison of robotic locomotion systems for truss structures. (L) Truss climbing robot system, based on work from [9]. $(R)$ Relative robotic system

Existing examples of robotic truss traversal focus on the local geometry of the framework - struts and nodes [9] [10]. These robots utilize a simple manipulator which is able to locomote along a strut, either through swinging [10], wheels [11] or with bidirectional gearing [9]. Two of these manipulators connected with a hip joint allow the robot to transfer from one strut to another, and therefore traverse threedimensional frameworks (Figure 2). In the case of Nigl, et al.[9], the robot also demonstrated traversal and basic reconfiguration, assembling and disassembling.

In both the framework construction and traversal robotics, however, the design objective is a general solution to the traversal problem, such that any framework geometry could be negotiated. This approach is appropriate when the goal is to traverse a structure such as the Eiffel Tower, where struts of varying length and nodes of various shape are employed. On the component level, however, designing the locomotion mechanisms around specific aspects of the geometry, or modifying the structures to interface better with the robot, has enabled an increase in the reliability of these robots. For Shady $3 \mathrm{~d}$ [10], the most robust locomotion example used a compliant system that assumed a $2.5 \mathrm{~cm}$ wide strut width, and with Nigl et al.[9] the struts were sheathed in bidirectional gearing in order to obtain the necessary position accuracy.

Digital cellular solids allow expanding this structural modification to encompass the design of the entire robot, due to two qualities of digital cellular solids: a geometry that can be defined by a translationally-invariant unit cell and parts with identical physical interfaces. The motivations for this expansion, which we will demonstrate in this paper, are:

1. Path Simplification: due to the periodicity of a digital cellular solid, three-dimensional traversal with a relative robot can be decomposed into a discrete set of motions (climbing, turning) that simply need to be repeated in order to reach any location/orientation in the structure.

2. Minimal Feedback: The robot uses the structure as an alignment mechanism, reducing the likelihood of failed grips. Locomotion along a strut, alternatively, requires either prior knowledge of the strut length [9], or sensors to inform the robot when it reaches a node and must navigate around it [10].

3. Reduced Mechanical Complexity: In addition to simplifying the motion planning and reducing the need for alignment feedback, the robot can also locomote with 5 DOFs. This is compared to [10], [11] and [9] for strut traversal robots.
However, disadvantages of this approach include the fact that the size of the robot scales with that of the unit cell of the lattice being constructed, while a truss robot only needs to be large enough to grip a strut and locomote around a node. Additionally, the interfaces between the robot and the structure depend on the shape of the struts and the nodes, while the overall design of the robot changes based on the lattice type (i.e. Kelvin, Octet, etc.)

We will detail a general strategy for designing a relative robot capable of traversing a CubOct lattice, and show an instantiation of this strategy that is designed to traverse a 76.2 $\mathrm{mm}$ pitch lattice. For the remaining sections, 'MOJO' will refer to this instantiation, while 'CubOct relative robot' will refer to the general class of robots to which MOJO belongs.

\section{METHODOLOGY}

\section{A. Lattice Design}

The structure used in this paper is a CubOct lattice, which is composed of vertex-connected octahedra connected in a cubic array. A single unit cell of this structure is referred to as a 'voxel', or volumetric pixel (Figure 3). The voxels used here have a lattice pitch L of $76.2 \mathrm{~mm}$ (3.0") and a strut length of $\mathrm{L} \sqrt{2} / 2$, or $53.88 \mathrm{~mm}$ (2.12"). The strut has a square cross section with a side length $\mathrm{L}=1.5 \mathrm{~mm}(0.056$ "). The parts are injection molded Zytel reinforced with $30 \%$ chopped glass fiber, and are joined using 0-80 screws and nuts.

\section{B. CubOct Relative Robot}

The CubOct relative robot requires two capabilities in order to traverse the lattice: it must be able to move forward in the direction it is facing (climbing), and it must be able to reorient itself within the lattice to change its facing (turning) (Figure 4). The symmetry of the framework can be used here to simplify the motions that produce these capabilities; for instance, by rotating about the cube diagonal (111)-axis [12], a robot oriented along one of the principal directions of the lattice can reorient itself along any of the other principal directions. We therefore propose a three mechanism layout for the CubOct relative robot: two identical arm mechanisms responsible for the gripping and translating, connected with an actuated hip that rotates about the cube diagonal axis.
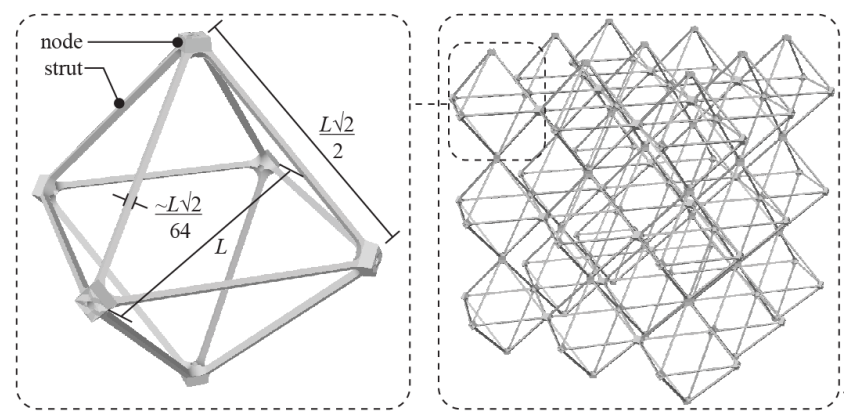

Figure 3: Octahedral voxel geometry and 3D lattice structure. $(L)$ Building block voxel, (R) $3 \times 3 \times 3$ cube of voxels. 


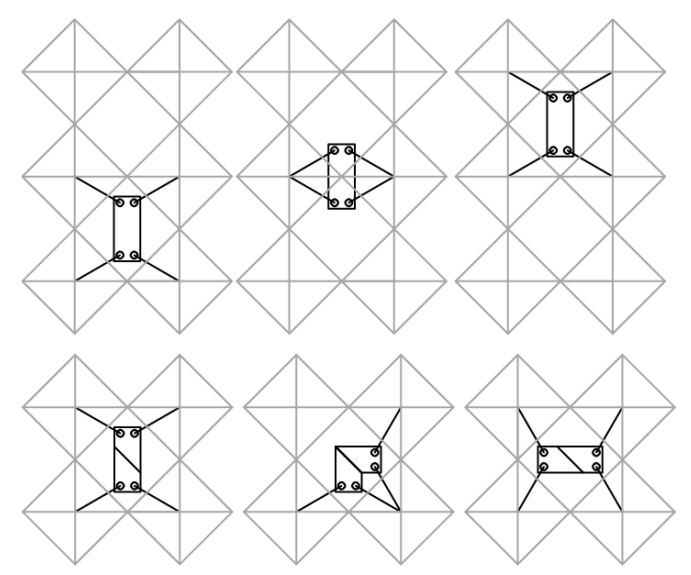

Figure 4: CubOct relative robot primary functionality. (Top) Climbing, (Bottom) Turning

In this layout, the arm mechanism must be able to reach three states: engaged outward, where it is gripping the structure and furthest from the hip, engaged inward, where it is gripping the structure and closest to the hip, and disengaged, where it is not touching the structure and free to move. In order to traverse the lattice, the distance between engaged outward and engaged inward must be a minimum of half a unit cell distance. If the two mechanisms are separated by half a unit cell distance as well, then the motion between the two engaged states is symmetric, which simplifies the path planning. Referring to the arm mechanism located above the hip as 'top' and the mechanism below the hip as 'bottom', the sequence of states required to produce motion from the center of one unit cell to the next is as follows:

\section{BOTTOM ENGAGED OUTWARD \\ 2. TOP ENGAGED OUTWARD \\ 3. BOTTOM DISENGAGED \\ 4. TOP ENGAGED INWARD \\ 5. BOTTOM ENGAGED INWARD \\ 6. TOP DISENGAGED \\ 7. GOTO 1.}

Combined with the arm mechanisms, the hip requires two states in order to be able to allow the robot to reorient itself within the structure: straight, where the two arm mechanisms are aligned along the same axis, and twisted, where the arm mechanisms are now aligned along two different principal axes. In order to reorient itself along a new principle axis, the sequence of states is as follows:
1. BOTTOM ENGAGED OUTWARD
2. HIP STRAIGHT
3. TOP ENGAGED OUTWARD
4. BOTTOM DISENGAGED
5. HIP TWISTED
6. BOTTOM ENGAGED OUTWARD
7. TOP DISENGAGED
8. GO TO 2.

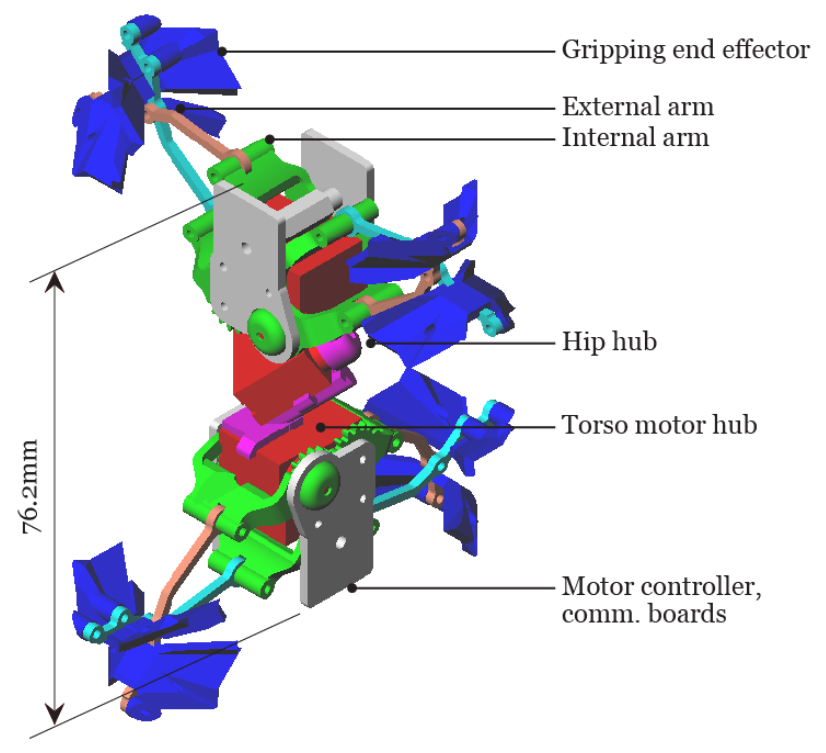

Figure 5: Overview of robot components and general dimensions.

This twisting corresponds to a 120 degree rotation about the cube diagonal axis, and by repeating three twists, the robot can rotate completely around in the unit cell.

\section{MOJO Mechanical Subsystems}

There are two mechanical subsystems for MOJO: the linkages that perform the engaging and disengaging motions for the arm mechanisms, and the end-effectors that interface between the linkages and the lattice (Figure 5). The arm linkage for MOJO is a pantograph mechanism, which allows it to grip onto the lattice and also sufficiently retract when disengaged. This linkage is actuated by two Hitec HS5035MG Servos, and is symmetric about the vertical axis- a gear ensures that the two sides of the arm mechanism set are kinematically connected (Figure 6).

The lengths of the bars for the arm linkages were chosen so that it could reach the required range of motion $(38.1 \mathrm{~mm})$ without overextending, and still retract without interfering with the structure. These dimensions were constrained by interference between the servo actuating the hip and the interior arms, which limited the maximum angle the interior arms could reach to 25 degrees from the vertical axis.

TABLE I.

ROBOT PHYSICAL PARAMETERS

\begin{tabular}{cl}
\hline Parameter & \multicolumn{1}{c}{ Properties } \\
\hline Mass & $\begin{array}{l}0.051 \mathrm{~kg} \text { (no batteries), 0.069kg } \\
\text { (with batteries) }\end{array}$ \\
Overall Dimensions & $76.2 \times 76.2 \times 76.2 \mathrm{~mm}$ \\
Servo Motor & $\begin{array}{l}\text { Hitec HS-5035HD; } \mathrm{m}=4.5 \mathrm{~g} \text {, Stall } \\
\text { torque @ } 4.8 \mathrm{~V}=0.8 \mathrm{~kg} * \mathrm{~cm}\end{array}$ \\
Exterior Arms & $1.5 \mathrm{~mm} 308$ stainless steel \\
3D Printed Parts & Interior arms, hips, end effectors \\
\hline
\end{tabular}




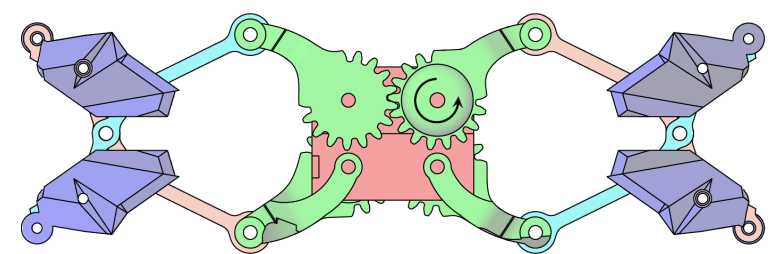

Figure 6: Arm set actuation. Both servos (red) drive a pair of geared inner arms (green), one set on the upper front and one set on the lower back. These then drive the passive outer arms (cyan and orange), which then actuate the gripping end effectors (blue).
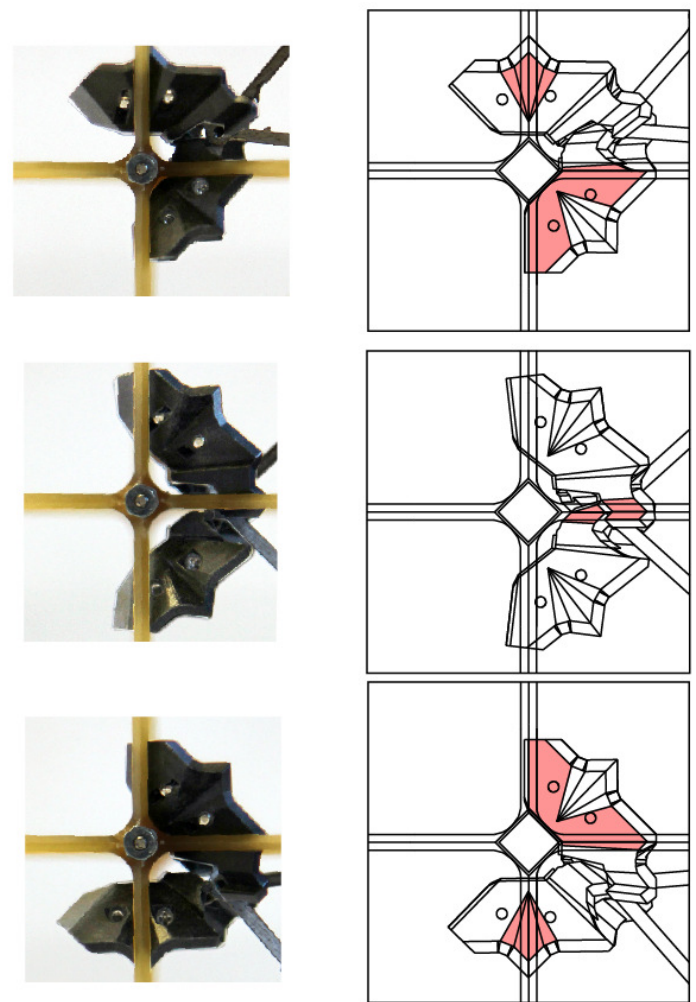

Figure 7: End effector and lattice interface. The main steps used to climb are shown with physical prototype $(L)$ and diagram with areas interfacing with lattice highlighted in red $(R)$.

The final dimensions of the mechanism and the maximum angle of the interior arms then allowed the calculation of the shape of the end-effectors (Figure 7). These large surfaces grip both the node and surrounding area of the structure, in order to provide reinforcement during climbing. The regularity of the physical dimensions of the structure allowed the introduction of a specific modification to the effectors; grooves corresponding to the location of the structure at the endpoints in the motion, which helped align the robot while switching between the extended states.

\section{MOJO Electrical Subsystem}

The electrical subsystem for MOJO consists of two parts: the control system which translates high-level commands to motor positions and senses power consumption through a current sensor, and the power system which boosts the input voltage to the operating voltage of the motors (Figure 8).

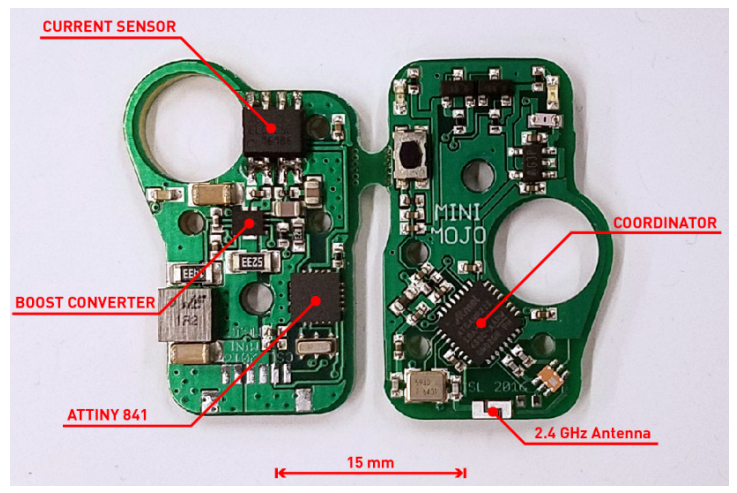

Figure 8: Board Layout. (L) Power board, x2 total on MOJO. (R) Communication/control board, xl total on MOJO.

The control system consists of an Atmel ATSAMR21 ARM Cortex-M0+ based 32-bit microcontroller/2.4GHz RF transceiver and two Atmel ATtiny841 8-bit microprocessors. The R21 routes commands and communicates with the two 841 slaves over an I2C bus. The 841's each control an arm set, with one 841 also controlling the hip servo, using three PWM channels controlled through an internal timer. The 841 's can also read an analog voltage value corresponding to the present current consumption from an Allegro Microsystems ACS712 Hall Effect-based current sensor.

The power system consists of a Texas Instruments TI61089 synchronous boost converter designed to step the typical operating voltage of a lithium-polymer battery, $3.7 \mathrm{~V}$, to $4.8 \mathrm{~V}$. The TI61089 was chosen because of its relatively small size and high efficiency; it is capable of delivering more than $90 \%$ power efficiency at an operating current of 2 A. On a full charge, the batteries last between 20-30 min. Assuming 20/s per cell, this results in an expected 60-90 cells capable with a single charge. Currently, that achieved range is between 10 to 20 cells, which is attributed to a sub-optimal arm path, which consumes more energy per cycle.

The boards housing the control and power systems double as mounting plates for the arm mechanism to the servo. This is achieved with a set of steel dowel rods which are epoxied into place and around which the arms pivot, and an additional bolt to prevent the mechanism from slipping.

\section{E. Testing MOJO}

In order to test our implementation of this CubOct relative robot, we performed three locomotion experiments with MOJO: climbing vertically, climbing horizontally, and turning. The difference between climbing vertically and horizontally is the orientation of the gravity vector and the robot; in the vertical climbing experiment, the gravity vector is aligned with the direction of motion and does not impact the alignment of the robot, but in the horizontal climbing experiment the gravity vector applies a torque to the robot, causing it to misalign. 


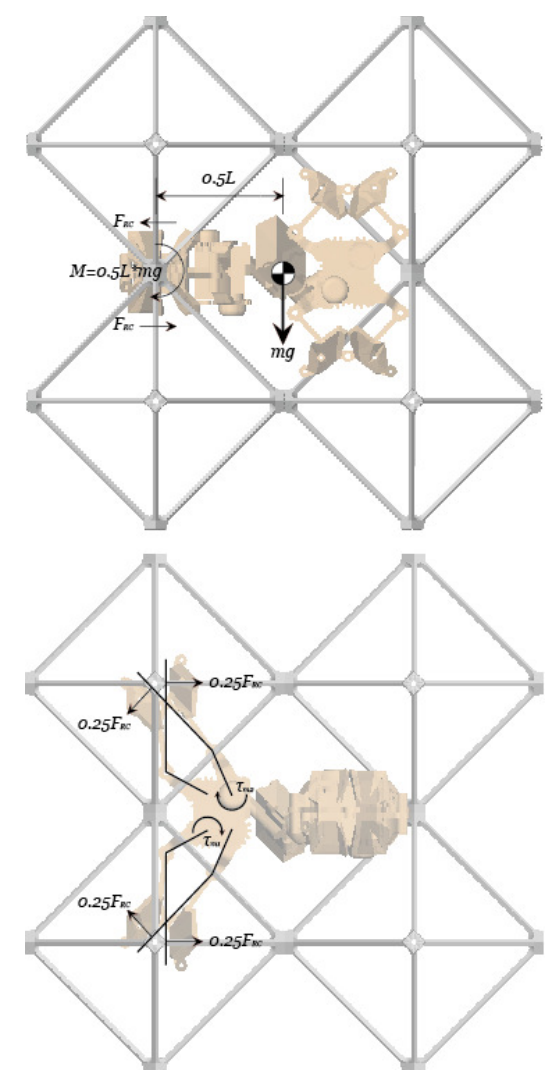

Figure 9: Free Body Diagram of horizontal climbing relating selfweight to motor torque. (Top) Side View (Bottom) Top view.

TABLE II. ROBOT TEST RESUlts

\begin{tabular}{cc}
\hline Parameter & Properties \\
\hline Climbing max speed (vertical) & 10 s/cell \\
Climbing success rate (vertical) & $100 \%$ \\
Climbing max speed (horiz.) & $10 \mathrm{~s} / \mathrm{cell}$ \\
Climbing success rate (horiz.) & $50 \%$ \\
Turning max speed & $5 \mathrm{~s} / \mathrm{turn}$ \\
Turning success rate & $67 \%$ \\
\hline
\end{tabular}

\section{EVALUATION}

In both locomotion experiments, the robot traversed two unit cells by transitioning between the motor coordinates corresponding to the movement states. The initial experiments attempted to directly transition between states without interpolation, but the arms could not move through the structure without intermediate positions to avoid interference with the structure. The final motion is an interpolation between key frames, performed in open loop, autonomously after an initializing command is sent. Combinations of maneuvers will be explored in further research.

Despite being nominally composed of the same set of motions, horizontal climbing displayed half the success rate of vertical. This was attributed to two causes: the direct transitions between the states did not sufficiently grip the structure during the intermediate movement, and the torque on the motors during the worst-case cantilever was near the limit of the stall torque for the servo. This indicates that openloop control may be best suited for environments where selfweight will not significantly alter the motion of the robot, such as microgravity.

The worst case horizontal climbing position is when MOJO is fully extended and only contacting the structure with a set of arms oriented perpendicular to the gravity vector. The torque from the cantilevered mass of the robot is applied through the kinematic chain of the arm mechanism to find the resulting back-torque on the servos (Figure 9). A torque of $0.33 \mathrm{~kg} \cdot \mathrm{cm}$ to the inner servo and $0.76 \mathrm{~kg} \cdot \mathrm{cm}$ to the outer. Since the stall torque of the servos is $0.8 \mathrm{~kg} \cdot \mathrm{cm}$, this backtorque is close enough to produce the observed misalignment. To alleviate this with the current robot design, we rotate the lattice 45 degrees along the horizontal axis, allowing the robot to traverse horizontally while reducing misalignment (Figure $10)$.

\section{CONCLUSION}

We described a methodology for designing the CubOct relative robot, which is specifically adapted to traversing the CubOct periodic lattice. We showed how the periodicity and symmetry of the structure provided opportunities to reduce the number of DOFs in the robot, simplify the mechanisms which compose the robot, and reduce the complexity of the path planning to actuate the mechanisms.

We applied this methodology with a robot design called MOJO, and tested it with three locomotion experiments: vertical climbing, horizontal climbing, and turning. MOJO displayed acceptable performance during vertical climbing and turning, but it exhibited misalignment during the horizontal motion. We attribute this to two factors: a path that insufficiently gripped the structure while transitioning between states, and an arm mechanism design that placed excess torque on the outer servos in the worst-case position.

The former factor can be addressed by finding paths that interpolate between multiple intermediate positions will ensure that the robot is in contact with the structure while locomoting. Integral to the evaluation of these more complex paths is the use of the on-board current sensors capable of measuring the motor torque at different points in the movement. By aiming for a current that indicates force is applied without approaching stall, the robot can use these sensors to find paths that maintain contact without wasting energy deforming the structure.

The latter factor can be addressed by finding iterations of the arm design that account for the forces experienced from horizontal climbing. These forces will be further exacerbated with the addition of batteries; two $350 \mathrm{mAh}$ batteries, one for each half of the robot, weighs $18 \mathrm{~g}$ total, and brings the backtorque on the outer servo during the worst-case cantilever up to $1 \mathrm{~kg} * \mathrm{~cm}$. A characterization of the effect of different linkage dimensions on the maximum torque will be necessary to find a mechanism that satisfies the physical requirements without exceeding the stall torque of the motors. 

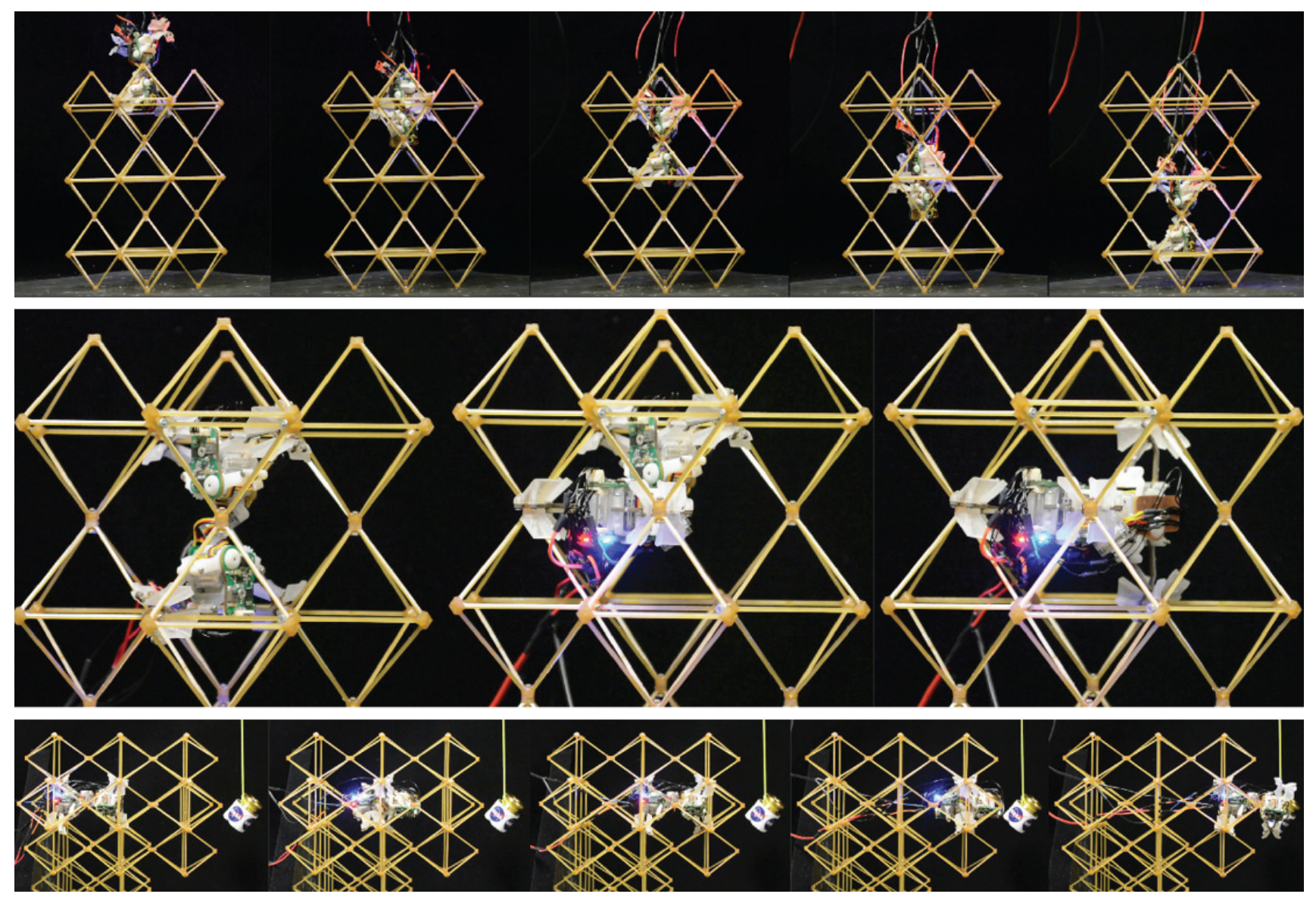

Figure 10: Demonstration of primary movements. (Top) Vertical Climbing in Z direction, (Middle) Turning from $Z$ to $X$ axis orientation, (Bottom) Horizontal Climbing in $X$ direction (with weighted marker to indicated orientation of gravity vector, in $Z$ direction).

\section{REFERENCES}

[1] K. C. Cheung and N. Gershenfeld, "Reversibly assembled cellular composite materials.," Science, vol. 341, no. 6151, pp. 1219-21, 2013.

[2] B. Jenett, K. C. Cheung, and S. Calisch, "Digital Morphing Wing: Active Wing Shaping Concept Using Composite Lattice-based Cellular Structures," Soft Robot., vol. 3, no. 3, 2016.

[3] B. Jenett, D. Cellucci, C. Gregg, and K. C. Cheung, "Meso-scale digital materials: modular, reconfigurable, lattice-based structures," in Manufacturing Science and Engineering Conference, 2016.

[4] T. a Schaedler, A. J. Jacobsen, and W. B. Carter, "Materials science. Toward lighter, stiffer materials.," Science, vol. 341, no. 6151, 2013.

[5] K. C. Cheung, "Digital Cellular Solids: reconfigurable composite materials," Massachusetts Institute of Technology, 2012.

[6] P. J. Staritz, S. Skaff, C. Urmson, and W. Whittaker, "Skyworker: A robot for assembly, inspection and maintenance of large scale orbital facilities," in
Proceedings - IEEE International Conference on Robotics and Automation, 2001, vol. 4.

[7] W. R. Doggett, "Robotic Assembly of Truss Structures for Space Systems and Future Research Plans," in IEEE Aerospace Conference, 2002.

[8] K. C. Galloway, R. Jois, and M. Yim, "Factory floor: A robotically reconfigurable construction platform," Proc. - IEEE Int. Conf. Robot. Autom. , 2010.

[9] F. Nigl, S. Li, J. E. Blum, and H. Lipson, "Structurereconfiguring robots: Autonomous truss reconfiguration and manipulation," IEEE Robot. Autom. Mag., vol. 20, no. 3, pp. 60-71, 2013.

[10] Y. Yoon and D. Rus, "Shady3D: A Robot that Climbs 3D Trusses," in IEEE International Conference on Robotics and Automation, 2007.

[11] C. Balaguer, A. Gimenez, and C. Abderrahim, "ROMA robots for inspection of steel based infrastructures," Ind. Robot An Int. J., vol. 29, no. 3, pp. 246-251, 2002.

[12] N. W. Ashcroft and N. D. Mermin, Solid State Physics, vol. 2, no. ISBN 0-471-92805-4. 1976 